\title{
DOSSIÊ
}

Sociologias, Porto Alegre, ano 5, no 9, jan/jun 2003, p. 64-104

\section{Reflexiones sobre una estrategia metodológica para el análisis de las protestas sociales}

ADRIAN SCRIBANO*

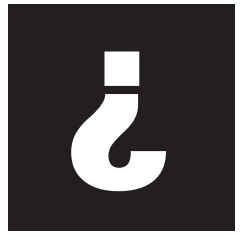

Cómo identificar, entender y explicar el sentido de las acciones colectivas? Es tal vez hoy una de las preguntas más desafiantes y conflictivas para la Teoría Social en general y la Metodología de las Ciencias Sociales en particular.

Desde una perspectiva teórica son diversas las opciones disponibles para interpretar las acciones colectivas y muchas de ellas implican la opción de una específica estrategia metodológica. En esta dirección, al intentar construir un diseño de investigación para el análisis empírico de las protestas sociales se hace evidente, de forma inmediata, algunas de las dificultades básicas de la producción de conocimiento en ciencias sociales.

Desde 1991 se han sucedido en Argentina una serie de protestas sociales que más allá de su orientación, contenido y eficacia han concentrado la atención de las autoridades, periodistas y analistas por su forma. Dichas protestas son los denominados cortes de ruta que consisten en obstaculizar el tráfico de vías de comunicación estratégicas. En todo el país los "Cortes de Ruta" son una forma de protesta que utilizan desde centros vecinales a colectivos de productores agropecuarios. Desde 1996 hemos venido trabajando tratando de explorar la "novedad" de sentido que estas acciones colectivas implicaban en el marco de las políticas de ajuste.

* Professor da Universidade Villa María, Córdoba, Argentina. Dr. 
Los primeros trabajos (Scribano, 1999b) realizados se orientaban directamente con la pretensión de enfatizar el rol jugado por la forma y los mensajes en los análisis de los cortes de ruta. Lugares desde donde la problemática de la visibilidad social de los actores emergía en relación con el de la constitución identitaria de los actores colectivos de las aludidas protestas. De esta manera, se ha mostrado cómo la lucha por la definición del sentido es una de las características de los cortes de ruta, o para ser precisos que existe en la constitución de dichas acciones colectivas el interés por la definición del sentido socialmente válido.

Además, en el contexto de la significatividad de la acción colectiva se ha podido observar cómo la constitución del juego visibilidad-invisibilidad opera de diversas maneras. Aludiendo así, a los efectos de los procesos de identidad colectiva que los grupos viven particularmente y que se cristaliza de manera especial en lo que se denominó densidad significativa de la acción colectiva. En conexión con lo anterior, se afirmó que las desigualdades sociales se vienen metamorfoseando, en una suerte de proceso de acumulación de situaciones de distribución diferencial e injusta de diversos recursos. Se intento así, mostrar cómo la pluralidad de desigualdades se presenta como punto de partida para la recuperación de la ciudadanía, como plataforma del reclamo de autonomía y solidaridad.

El correr del tiempo y la extensión de la utilización del "corte de la circulación" como instrumento de protesta planteo la necesidad de preguntarse por los modos de producción, reproducción y apropiación de dicho "instrumento" que se verifican en los colectivos que protestan.

Desde 1998 (aproximadamente) se ha podido observar como los cortes de ruta han sido utilizados por actores que proviniendo de los más variados orígenes confluyen en lo que se puede llamar la "protesta del sector agropecuario". En este sentido se comenzó una investigación sobre el Tractorazo en la provincia de Córdoba, que consintió en "cortes" cuyo símbolo y herramienta principal fue el tractor cruzado en la ruta. 
Sociologias, Porto Alegre, ano 5, no 9, jan/jun 2003, p. 64-104

Al explorar el contexto de surgimiento de estos cortes aparecieron desde los factores climáticos pasando por la intrínseca variabilidad temporal del sector y llegando a los numerosos impuestos que se deben pagar. De este modo se constato que el "agro" cordobés se inscribe hoy en redes conflictuales locales y nacionales que trascienden las organizaciones de productores que aparecen en la convocatoria de la protesta. Entre otros hallazgos se pueden destacar los siguientes como los más importantes: a) al igual que otro tipos de corte aquí también jugo el complejo de significado entre visibilidad/invisibilidad; b) se manifestó con fuerza el aprendizaje social respecto a la forma de protesta debido a que ya se apreciaba que los actores consideraban al corte como una herramienta de lucha; c) la pluralidad de posiciones y condiciones de clase se mantenía al igual que en los otras cortes pero se visualizaba la presencia de organizaciones previas con alto grado de institucionalidad muchas veces con intereses contrarios; d) en este contexto se pudo observar la disolución de la representatividad sindical y social en el sector y e) se percibió la tendencia hoy acentuada de "desconexión" con los canales políticos formales.

En ese contexto, desde el 2000 se exploran respecto a la protesta social en general, las consecuencias para la política, la constitución de espacios públicos y la emergencia de nuevas "formas" de derechos que los conflictos y los actores que cortan las rutas pueden tener (Scribano, 2000 a; 2000b).

En el marco de las investigaciones reseñadas, este trabajo tiene por objetivo mostrar y discutir una de las estrategias usada para investigar "Cortes de Ruta" en la Argentina en tanto forma particular de protesta social.

Para lograr el objetivo planteado se ha seleccionado la siguiente camino argumentativo: a) sintetizar los supuestos epistémicos básicos del abordaje utilizado; b) reseñar el instrumental teórico construido; c) describir las técnicas de obtención de información y d) reflexionar respecto a las dificultades encontradas y a las potencialidades detectadas. 


\section{Sinstesis de los supuestos epistemicos del analisis de la protesta}

Es muy difícil reseñar en pocas páginas los supuestos epistemológicos que subyacen a una investigación empírica, dado que estos se van constituyendo en una trama abigarrada donde se cruzan herencias teóricas, formación académica, oficio de investigador, inclusión en el campo disciplinar y contexto social. Visto de esta manera, una explicitación de dichos supuesto llevaría a la auto-aplicación de un socio-análisis (sensu Bourdieu) que nos alejaría de los objetivos de este trabajo.

Sin embrago, aclarado lo anterior, es posible objetivar, al menos parcialmente, algunos de los supuestos del continuo que va desde los supuestos ontológicos a la selección de los conceptos operacionales de la investigación que se esta analizando.

Es preciso señalar que, desde un punto de vista muy "macro", la opción fundamental es la de entender la tarea científica desde un contexto post-empirista que propicia un posicionamiento pluriparadigmático, tanto en lo teórico como en lo metodológico. Esto se cualifica y particulariza aclarando que se ha optado por un camino de articulación entre realismo, hermenéutica y teoría crítica, muy cercano aunque no idéntico, al emprendido por el realismo critico ingles en los años 70, especialmente en la forma que lo ha expuesto William Outhwaite.

Realizadas estas aclaraciones y especificando su alcance en el campo discursivo de los estudios de acciones colectivas, se puede afirmar que genéricamente se han seguido los siguientes lineamentos en tanto supuestos epistémicos:

1. afirmar la disolución de un enfoque dualista respecto a la relación entre objetivismo y subjetivismo entendida en términos "aporéticos";

2. posicionarse más allá de la discusión respecto a lo micro y a lo macro como irreconciliables niveles de análisis; 
Sociologias, Porto Alegre, ano 5, no 9, jan/jun 2003, p. 64-104

3. enfatizar la importancia del giro lingüístico y de la impronta herme-néutica, como así también, suscribir la superación de la falsa contradi-cción y confrontación entre enfoques cualitativos y cuantitativos;

4. partir de la reformulación del lugar y rol de la percepción en la observación sociológica, como resultado de la aceptación de una nueva manera de entender las relaciones entre términos teóricos y observacionales en Ciencias Sociales.

Estos presupuestos generales se complementan con otros de carácter más específicos en el campo teórico, ontológico y epistémico, entendiendo que toda tarea de investigación implica la aplicación e interrelación continua de dichos supuestos. (Scribano, 1998a) Los presupuestos específicos pueden sintetizarse de la siguiente manera:

\section{Subjetividad e Identidad}

En primer lugar, se opta por una reconstrucción de la noción de sujeto que implicaba la idea de actor social. En este sentido, aparece la transformación de la imagen de un individuo todo poderoso que domina el mundo natural y social. Este desdibujamiento de la idea "moderna" de sujeto implica también la disolución de un sujeto como constructor de la realidad social recortada al talle de su propia individualidad.

Es en esta dirección que emerge una noción de subjetividad que encuentra el fundamento para la confianza y el reconocimiento mutuo en el marco de una revalorización del cuerpo en tanto modo primario de reconocimiento y autoreconocimiento. La subjetividad deviene un proceso de auto-construcción de la identidad. En el duro itinerario para hacerse individuo el ser humano encuentra la posibilidad de superación de las fronteras de la sociedad compleja en tanto mecanismos de control. La transformación de la intimidad promueve a un individuo que responde a una imagen de sujeto que va más allá de los dualismos, mente-cuerpo, cultura-naturaleza y mundo interno-mundo externo. El ser humano es una 
subjetividad en el diálogo cotidiano con los "otros". Por lo que, se disuelve la teoría social pensada desde la reclusión de la metáfora de la conciencia como espejo y la imagen moderna de sujeto. Suponiendo que el sujeto se constituye en la experiencia de la identidad encarnada en un cuerpo y en el proceso de reconocimiento de y con los otros.

2 Hacia una sociología de la "experiencia"

Los cambios producidos en las últimas tres décadas muestran nuevos caminos teoréticos para las ciencias sociales, y los rasgos reflexivos de la Teoría Social establecen nuevos tipos de respuesta a los desafíos de los problemas sociales. Los procesos identitarios (individuales y colectivos) se afirman como uno de los más profundos cambios de este contexto; acentuándose el desplazamiento de la teoría sociológica hacia las interconexiones entre lo subjetivo y las condiciones "estructurales" redefinidas fuera de un marco aporético de análisis. Por esto, estamos de frente a una particular sociología de la experiencia humana. La acción social es un espacio multipolar; donde los ejes de lo individual y lo colectivo tienen una relación de profunda interconexión. La construcción del proceso de la identidad individual obtiene nuevas dimensiones en este espacio. La tarea de auto-reconocimiento produce un dominio compartido el mecanismo de reconocimiento de los otros. Los rasgos personales de la acción social logran mayores dimensiones en la comprensión sociológica dado este contexto dialógico. La experiencia vivida "con" y "de" los otros en el marco de las transformaciones del si-mismo genera un campo de análisis diferencial en el conocimiento sociológico. Los dilemas de la sociedad compleja, libertad y sobre-control, la capacidad de otorgar sentido y las posibilidades de autodestrucción, etc., hacen desplazar, más y más, el conocimiento sociológico hacia el si-mismo y su campo de relaciones con los otros. Los desafíos de nuestras sociedades exclusoras y neo-coloniales, hacen pensar en las acciones colectivas como el espacio relacional donde 
Sociologias, Porto Alegre, ano 5, no 9, jan/jun 2003, p. 64-104

las conexiones aludidas se instancian muy particularmente.

3 Más allá de la linealidad

Se parte del rechazo al uso de un esquema lineal de causa-efecto. La postulación del proceso de producción y reproducción social implica la disolución de los enfoques que conectan solamente causa y efecto linealmente y de un modo teleológico. La era de la información hace surgir un mundo simultáneo, donde las nociones de tiempo y espacio son reconstruidas en sentidos diversos a los modos de comprensión precedentes. La investigación social encuentra, de esta manera, nuevos tipos de relaciones sociales, por lo cual la comprensión sociológica necesita un especial enfoque no lineal respecto a la coordinación de la acción. Esto se afianza sí se piensa que la utilización de la metáfora del círculo y el uso de lo paradojal como recurso interpretativo involucran una manera distinta de pensar la relación entre eventos. En el contexto de un mundo instantáneo, se manifiesta para el conocimiento sociológico el desafío de comprender la manera diferente de presentarse que adquiere lo constante y lo variable como rasgo de la realidad. Los mecanismos de producción y reproducción hacen emerger con fuerza el dominio recursivo de la acción social donde tienen lugar la compleja relación entre los rasgos constantes y variables del mundo social. Las sociedades complejas cancelan, al menos parcialmente, la idea de sucesión temporal antes-después y en este sentido la Teoría Social tiene la necesidad de un esquema diferente para entender dicha conexión. Esto se profundiza en esta suerte de cruce entre post-modernidad y colonia que evidencian nuestros procesos de estructuración, donde las tensiones externo-interno, nosotros-ellos, clase-funsión, entre otras, aparecen como rasgos sobresalientes.

\section{La Relacionalidad}

En la actualidad la Teoría Social tiene en la relacionalidad una de sus características centrales. La práctica social se manifiesta como un logro 
procesual de la intersubjetividad. Este tipo de esquema de significación sociológico implica el fin de una imagen antropocéntrica del conocimiento. Objeto, sujeto y sus relaciones son los elementos del conocimiento con el mismo nivel de importancia en el proceso de comprensión de la realidad. Más allá del objeto y del sujeto, más allá de la aceptación de la existencia externa a la conciencia de las cosas o como construcción de una esfera cognitiva, el conocimiento aparece como proceso. La procesualidad muestra la relacionalidad, no solamente desde un punto de vista metodológico, además, como una fundamental pero no substancial, central pero no exclusiva característica del conocimiento. El conocimiento tiene lugar en el propio proceso donde objeto y sujeto se auto-develan y entienden, el mismo proceso de reconocimiento entre objeto y sujeto brinda la posibilidad de conocer. Sobre esto voleveremos más adelante.

\section{Sociología de los "Dilemas"}

En el ámbito de la "matriz moderna" del pensamiento sociológico afirmar dos interpretaciones para el mismo fenómeno era considerado inadmisible. Los dilemas aparecen en un tipo "nuevo" de sociología donde conceptos tales como dialéctica y contradicción se redefinen y alcanzan un puesto de relevancia en las interpretaciones sociológicas. Las consecuencias locales del cambio global, la potencialidad de autodestrucción que anida en el gran avance tecnológico, la oportunidad humana de otorgar sentido al mundo en el contexto de una sobre-controlada situación mundial, las resistencias mundiales ante el dominio neoliberal, ejemplifican cómo los fenómenos de "dos caras" pueden impactar en los intereses de la teoría social. En la actualidad el movimiento de la vida social tiene un rostro de doble lado. Esta paradoja es una de las más particulares características de las sociedades complejas y de las sociedades de los márgenes como las nuestras, y presiona por la constitución de un adecuado marco conceptual de análisis. La vida cotidiana es construida como 
Sociologias, Porto Alegre, ano 5, no 9, jan/jun 2003, p. 64-104

un juego entre elección y determinación, y hace emerger los dilemas aludidos. Consecuentemente surge una Teoría Social interesada en hacer posible la comprensión de fenómenos sociales contradictorios y paradójicos.

\section{Dialéctica, Relacionalidad e Indeterminación}

Finalmente, en una apretada síntesis, los trabajos que hemos realizados sobre acción colectiva parten del supuesto que nuestro conocimiento de la realidad social es posible de ser producido si lo entendemos en el cruce de dialéctica, relacionalidad, e indeterminación.

La utilización de la noción de dialéctica involucra a la vez rasgos ontológicos de la realidad social, presupuestos epistémicos para su comprensión y supuestos teóricos de las ciencias sociales.

En principio la noción de dialéctica que se ha utilizado aquí nos hace pensar en tres posibles significados: en primer lugar se puede identificar con una visión procesual y relacional de los fenómenos que estructuran el mundo social; en segundo lugar indica un proceso propedéutico para el conocimiento de lo social, finalmente, (de un modo menos fuerte) señala los momentos de articulación de los fenómenos acaecidos en la estructuración social. El primer sentido aborda la faceta de constitución del mundo social que ha sido generalmente acotada, pero no resuelta, en la dicotomía diacrónico/sincrónico. Es decir, lo social no se pude agotar en el saber sobre la simultaneidad o en el saber sobre el devenir, para ello hace falta incorporar una mirada que permita captar lo que hay de simultáneo y lo que hay de cambio en el mundo social. Lo dialéctico es, así, un rasgo de lo social que involucra sus momentos de cambio y de permanencia cristalizado en lo que es el proceso de relacionalidad de los agentes y las acciones sociales. En esta dirección la dialéctica de la estructura y de la praxis puede ser identificada con las características básicas de relación entre agente y estructura, entre agentes y entre acciones, que otorga a la realidad social esa cualidad de aparecer como simultanea y cambiante a la vez. Este rasgo 
de la constitución social conduce a preguntarse por cómo emergen los momentos de producción y reproducción de la realidad social. De este forma se pude comprender que sí se observa detenidamente tanto la estructura y la praxis son ambas medios y productos de la agencia humana. Por lo tanto se podrá apreciar como lo dialéctico es un camino cognoscitivo y argumentativo para adentrarse en una realidad social que se presenta como compleja e indeterminada. Es decir, dialéctico significa aquí el modo por el cual nos introducimos al conocimiento de una realidad que es simultánea y cambiante, producida y reproducida por los agentes. Es buscar la relacionalidad y la procesualidad, otorgarle un sentido que pueda ser comprendido en una dirección muy cercana con el trabajo de interpretación propuesto como tarea hermenéutica. Así, la dialéctica es el reconocimiento de las voces que emergen múltiples y complejas pero posibles de ser traídas a diálogo en una racionalidad procedimental. En este sentido la dialéctica es argumentación racional y es una forma de conocimiento y supuesto epistémico del conocimiento científico de lo social. Pero también se sugiere un tercer significado, que si bien es más "débil" que los anteriores es el más directamente usado en la elaboración de teoría en ciencias sociales, es el que indica que los procesos de articulación entre fenómenos no pueden ser identificados con los rasgos de esos fenómenos ni con los de los agentes, ni con los de la agencia. Justamente es el mismo momento de articulación que debe ser conceptualizando teniendo en cuenta los rasgos de aquello que se articula, que provee de una nueva realidad no identificable con las que le preceden.

Desde aquí, la práctica social se manifiesta como un logro procesual de la intersubjetividad. De acuerdo a esto, como se afirmó ya, la procesualidad del conocimiento es uno de los resultados más importantes de la sociedad y de la comprensión sociológica.

Desde lo afirmado se puede entender que las ciencias sociales son un esfuerzo de interpretación de la indeterminación, unas ciencias de un 
Sociologias, Porto Alegre, ano 5, no 9, jan/jun 2003, p. 64-104

mundo indeterminado. El intento es ir más allá de los significados primarios de dicha indeterminación, es decir, traspasar de modo sistemático la concepción de que nuestra tarea es siempre una acción sin determinar. Sea que esto se entienda como radicalmente interpretable, sea que se entienda en relación a la medición "inexacta" del mundo social.

La indeterminación no se relaciona ni con el determinismo ni con el indeterminismo de la traducción, como así tampoco con su uso en la física cuántica. Es un rasgo ontológico de la realidad social. Como afirma Bohman, Sí los agentes toman conciencia de y cambian las condiciones bajo las que ellos obran, no hay un factor o un set de factores que puedan completa o determinadamente explicar un evento o acción social (Bohman, 1994, p. 3). La indeterminación no consiste en la imposibilidad e incomprensión, sino que está relacionada con el carácter reflexivo del conocimiento.

La indeterminación opera bajo el supuesto de que estamos frente a sistemas abiertos y que el mundo es estructurado, diferencial y cambiante. Estos rasgos marcan la necesidad de partir de la aceptación de la inclusión en campo observacional de quien observa. La procesualidad es un momento del conocer la indeterminación radical de lo que se ha objetivado y marca el camino para una dialéctica que recupere la ruptura y la conquista con lo que se presenta como objetivo.

\section{Conocimiento sociológico y Reflexividad del conocimiento}

En el contexto de lo anterior, se sostiene la relación entre conocimiento y reflexividad en dos direcciones. En primer lugar, respecto a la aceptación de la reflexividad institucional de la sociología como un producto de la modernidad; en segundo lugar, en tanto su reconocimiento como una capacidad de la acción humana, esto es, la acción de auto-percepción y la posibilidad de "dar cuenta" de narrar y narrase que tiene el sujeto. La reflexividad implica la auto-capacidad del individuo de tomar su propia acción como un objeto analítico en el medio particular que implica su 
discurso sobre si mismo. Dado este contexto crece la necesidad de extender las conclusiones de los análisis de la reflexividad a la propia manera de ver y hacer sociología. Los mecanismos de reproducción del campo académico ya no se pueden desligar de la misma reflexión sobre la sociedad. La reflexividad como fenómeno revela la necesidad de una práctica sociológica auto-analizada encaminada a entender el proceso de construcción social de las discusiones en teoría social. Como así también, implica la urgencia en reparar en el entramado significativo que supone el construir conocimiento científico sobre el mundo social en tanto agente y en tanto científico social.

\section{Filosofía social, conocimiento sociológico y práctica social}

En el contexto reflexivo anterior se puede observar un particular regreso a las preguntas de la "filosofía social". Por esta vía, se sostiene que las consecuencias del análisis sociológico producen afirmaciones evaluativas respecto a la "vida buena" y el bienestar social. Dada la transformación de la filosofía de la historia y la disolución de las ideas teleológicas del futuro, no existe espacio para la fijación de un orden social "objetivamente bueno" como final de la historia humana. De todas maneras, podemos tratar de entender las posibilidades de emancipación desde los resultados del análisis producido por las Ciencias Sociales. Los logros de las Ciencias Sociales son producidos en el marco de una dialéctica entre valores y hechos; y contienen en todo momento una específica imagen de la sociedad. Así aparecen las clásicas preguntas respecto a la "vida buena" que desafían a las Ciencias Sociales a la búsqueda del cómo es posible encontrar una vida cada vez más humana para los seres humanos.

Esta apretada síntesis de los supuestos epistémicos que están por debajo de la investigación de la acción colectiva nos permite introducirnos a los nudos más sobresalientes de la construcción teórica realizada a tales fines. 
Sociologias, Porto Alegre, ano 5, no 9, jan/jun 2003, p. 64-104

\section{Principales opciones teoricas}

En el marco de los supuestos reseñados y dado el interés de conectar la teoría sobre movimientos sociales con los análisis de la protesta, se han usado como punto de partida para la reflexión las ideas de Alberto Melucci. Del contexto de su amplia producción se puede extraer el siguiente esquema analítico para comprender la acción de los movimientos, que se caracteriza por los siguiente elementos: a) Desde un punto de vista "epistemológico", se puede suponer que en el intento de analizar los movimientos hay un fuerte cariz cognitivo, dado que ésta acción parece construida como relación entre un objeto y un sujeto y que los dos producen representaciones sobre la realidad. Esto es, tanto los actores de los movimientos construyen su percepción sobre si mismos, como el investigador construye una representación sobre los movimientos como acción colectiva, lo que conduce a entender los grados de adecuación posibles entre lo que conceptualmente se identifique como acción colectiva y lo que esta sea en "la realidad" desde la perspectiva de los sujetos que actúan; b) Las nociones de "sentido" y "límites del sistema" surgen como característica de una acción cotidiana que desafía la misma complejidad del sistema y también permite captar la emisión de las "señales" de la acción colectiva como marca de la situación conflictual del sistema; c) Los aportes de Melucci permiten comprender también cómo la relación entre identidad y diferencia en el interno de los movimientos es un elemento importante del propio mensaje que encarnan. Identidad y diferencia que apuntan a sostener una mirada más cauta respecto a la relación entre el proceso de constitución de identidad personal (demandas de subjetividad) e identidad colectiva (demandas de visibilidad) (Scribano, 1999c).

De estos tres elementos conceptuales básicos, que emergen de una relectura de las ideas de Melucci se puede extraer respecto a nuestro interés principal algunas pistas conceptuales. Los movimientos en tanto acciones 
colectivas se relacionan directamente con un "campo conflictual", pueden implicar significados diversos y que las construcciones conceptuales no agotan ni coinciden con el fenómeno concreto de acción colectiva. Para avanzar en nuestra propuesta necesitamos diferenciar y aclarar de manera satisfactoria a los movimientos sociales como una de las manifestaciones posibles de la acción colectiva pero teniendo presente que no es la única forma que esta última puede adoptar. Por lo que, decidimos realizar una tarea de redefinición de los conceptos fundamentales ligados a la acción colectiva bajo el supuesto básico de que los Movimientos no agotan todas las posibilidades de acción colectiva y además de que algunos de los rasgos que hoy se conocen de los Movimientos sirven para el análisis de las aludidas situaciones de Acción Colectiva y en especial de las Protestas.

En este sentido y para mejorar nuestra comprensión de las acciones colectivas y acrecentar el valor heurístico del concepto es necesario distinguir los siguientes aspectos: a) La relación y la diferencia entre Conflicto, Protesta y Accion Colectiva; b) Asumir algunos de los más importantes legados de lo que hoy conocemos sobre Movimientos Sociales; c) Re-leer los procesos de constitución social a la luz de lo que se ha aprehendido de las historias de estructuración de los campos de lucha sociales. En principio esto trae las siguientes consecuencias:

1. Distinguir entre los componentes de las "condiciones de la acción colectiva", los conflictos que la originan, la emergencia y manifestación de los conflictos y los modos de reproducción de las aludidas condiciones.

2. Asumir que las acciones colectivas son a la vez el resultado de otras acciones colectivas e individuales, y que producen también otras acciones colectivas. Aceptando que las acciones colectivas involucran momentos de latencia y de manifestación, haciendo referencia esto a su potencial o manifiesta visibilidad.

3. Se debe tener presente también, que los conflictos se desplazan y 
Sociologias, Porto Alegre, ano 5, no 9, jan/jun 2003, p. 64-104

adquieren Significados diversos en el mismo proceso de constitución de la acción colectiva.

4. Es necesario reparar en que las Manifestaciones y/o Emergencia de las acciones colectivas asumen un carácter precario pero estructurado y que por lo tanto se puede "periodizar" su aparición y su disolución.

5. Finalmente, se debe asumir que los agentes modelizan sus relaciones en conexión a el desplazamiento de los significados de los conflictos, lo que trae aparejado que se deba estar alerta para aceptar Paradojas y Contradicciones en las acciones colectivas.

Dadas las características de este trabajo nos concentraremos en un problematización provisoria sobre la relación y la diferencia entre Conflicto, Protesta y Accion Colectiva.

Existen diversas maneras de definir la noción de conflicto, en esto también se ha retomado aquí el enfoque de Melucci al respecto. Si bien él tuvo un proceso de cambio sobre su opinión respecto a la noción misma de conflicto, éste ha sido para él siempre el elemento central que permite discutir las acciones colectivas y más especialmente la conformación de los movimientos sociales. En este contexto, conflicto se define como aquella relación de dos (o más) actores sociales que luchan por el control de recursos a los cuales ambos le asignan un valor (Melucci, 1984, p. 423) Los recursos pueden ser de carácter material y/o simbólico y las valoraciones no tienen por que ser posibles de ser evaluadas de la misma manera. Para Melucci para que exista un Movimiento deben darse además otras dos características, la solidaridad y la ruptura. Siempre teniendo presente que los movimientos no agotan los tipos de acciones colectivas y que ellos mismos pueden albergar un cruce entre diferentes tipos de esas acciones, es necesario precisar que se entenderá por acción colectiva, ya que este es nuestro interés particular.

En principio, se puede afirmar que la acción colectiva es "considerada como la resultante de metas, recursos y límites" que ponen en juego los 
actores sociales; esta es entonces, el producto de orientaciones de la acción común en el marco de un campo de oportunidades y restricciones. En este contexto, Melucci afirma que, los individuos actúan colectivamente construyendo su acción por la definición, en términos cognitivos, de las posibilidades y limites (de su acción), al mismo tiempo que interactúan en orden a 'organizar' sus conductas comunes (Melucci, 1989, p. 25). Por lo que, la acción colectiva involucra interacción, negociación y conflicto, siendo este último el marco general necesario para que emerja dicha acción.

Por esta vía se hace evidente que para poder estudiar acciones colectivas es preciso analizar el campo de conflictos donde esta tiene lugar, y que la protesta es parte de dicha acción colectiva pero que no la agota. Dados nuestros intereses particulares es conveniente que re-utilizando y re-definiendo estos conceptos aclaremos detenidamente cuales han sido son nuestros puntos de partida en el análisis de los cortes de ruta en tanto protestas sociales.

En un conflicto entran en juego intereses, valoraciones simbólicas, actores y acciones diversas, en esta dirección se propone la noción de redes de conflictos (sobre la que enseguida se volverá) como un elemento clave para la comprensión de las protestas.

En este punto conviene aclarar previamente que, nuevamente, aparecen las ideas de Melucci sobre identidad colectiva como plataforma conceptual que permite repensar los cortes de ruta como protestas sociales en el marco de una teoría de la acción colectiva.

Luego de subrayar la imposibilidad de una metafísica de la acción colectiva y en el contexto de su análisis de la acción Melucci propone uno de sus conceptos más originales, la noción de identidad colectiva. Iniciando una crítica de la visión de los movimientos como un actor unitario, se señala que ésta unidad debe ser vista como el resultado del intercambio, la negociación, la decisión y conflicto. Por lo que, la construcción permanente de un "nosotros" donde reconocerse y ser reconocido es un punto clave 
Sociologias, Porto Alegre, ano 5, no 9, jan/jun 2003, p. 64-104

en el proceso de constitución de la identidad colectiva. Pero teniendo presente que los procesos que a través de los cuales es obtenida la unidad no son inmediatamente visibles, y cómo los actores tienden a enfatizar su carácter tenue y construido (Melucci, 1992b, p. 243). Este proceso implica una articulación entre metas, campo de acción, definición co-producida de la situación y negociación, es decir, es un proceso relacional por el cual emerge la identidad colectiva. Los individuos actúan colectivamente encontrándose en medio de un sistema multipolar constituido por tres ejes que se articulan y relacionan interdependientemente en una tensión mutua. Los individuos contribuyen a la formación de un más o menos estable "nosotros" por medio de volver común y laboriosamente, negociaciones y ajustes de al menos tres orientaciones: las metas de sus acciones; los medios que deben ser utilizados y del ambiente dentro del cual sus acciones tienen lugar (Melucci, 1989, p. 26). En este proceso de construcción de la acción colectiva emerge la identidad colectiva que tiene, considerada en cuanto proceso mismo, tres dimensiones: metas, sentido y ambiente. Dimensiones que se ponen en relación por medio de la formulación de un esquema de referencia cognitivo, poniendo así en funcionamiento una estrecha interrelación entre los actores que se comunican, negocian, toman decisiones y proveen de "inversiones" emocionales.

La identidad se presenta como una construcción que puede adoptar diversos grados de estabilidad o variabilidad, de integración o fragmentación. En este marco, Melucci afirma que la identidad colectiva "es una compartida e interactiva definición producida por varios individuos que inter-actúan quienes se implican con las orientaciones de sus acciones, tanto como con el campo de oportunidades y restricciones en el cual la acción tiene lugar" (Melucci, 1989, p. 34).

En este contexto, la identidad colectiva, como fenómeno que emerge de la negociación y de los conflictos, tiene la particularidad de no poder ser definida nunca de modo absoluto, es un proceso en si misma. 
De esta manera, una de las apuestas teóricas centrales de nuestros trabajos se debe contextualizar en la revisión de las nociones arriba aludidas de modo tal que permita operar sobre el "lado oscuro" de las nuevas formas de protesta, a saber, la constitución de "nuevas" prácticas y "nuevos" actores. En este sentido se sugiere que en los cortes en tanto protestas, (es decir modos de acción colectiva que no debe identificarse solamente con la acción de un movimiento) se produce la construcción de identidades colectivas fragmentarias y transitorias.

Hasta aquí, la relectura de la teoría de la identidad colectiva que se puede encontrar en los estudios de movimientos sociales, veamos ahora las construcciones teóricas que profundizan y particularizan una percepción de los cortes de ruta en especial y de las protestas en general.

Dicho giddensianamente, este aquí y ahora se instancia en el contexto de su estructuración y se trasciende en los significados de su presentificación. La protesta es en este sentido un mensaje a interpretar cuya forma es, en muchos casos, "el" mensaje.

Por lo tanto, desde los cánticos, pasando por las pancartas hasta la misma acción nos desafían a re-nombrarlos desde la compleja significación que involucra su evanescencia e indeterminación.

Sin duda alguna, el análisis de la acción colectiva involucra en tanto tal tres niveles de análisis: su conflictividad, su estructuración temporoespacial y sus modos de expresividad.

Como ya afirmáramos, por lo general toda acción colectiva que deviene protesta social implica la existencia de uno o más conflictos. Dichos conflictos pueden ser definidos en su cualidad más primaria y ontológica como el resultado de la diversidad de valoraciones que tienen dos o más agentes sobre un bien que ellos evalúan como importante. Dicha importancia puede provenir de la cualidad del bien en relación a la reproducción material de los agentes, de su peso simbólico o de otros mecanismos de constitución de la realidad social que transforme a la 
Sociologias, Porto Alegre, ano 5, no 9, jan/jun 2003, p. 64-104

producción acumulación y distribución de los bienes aludidos en un problema colectivo. En este sentido, la protesta social es precedida y presidida por situaciones conflictivas. Al conjunto de estos conflictos se los puede denominar como Redes de Conflictos.

Las Redes de Conflictos que preceden y operan como trasfondo de las Protestas actúan en el tiempo reconvirtiendo y redefiniendo las posiciones de los agentes y el sentido de las acciones.

Es en este sentido, que la protesta puede ser entendida si se analiza como la interconexión de diversos "momentos" de movilización que se generan y giran en torno a redes de conflictos, pero que no se agota en la sola manifestación de la acción colectiva, sino por el contrario están fuertemente relacionadas también con sus períodos de latencia.

En la dirección arriba señalada, en el análisis de la protesta hay que tener presente siempre a las Redes de Conflicto que a ella se conectan, como aquello que da forma a las "Condiciones de la Acción Colectiva". Es importante subrayar que lo que aquí se denomina red de conflictos, está constituida a su vez por relaciones entre actores que implican la referencia a redes de conflicto anteriores conectadas entre si. Es en este sentido, que en otro lugar (Scribano, 1999b),

se ha propuesto entender a los efectos del ajuste como trama que origina las expresiones de conflicto que implican los cortes, como la manifestación de dichos conflictos, orientada a obtener visibilidad cuyo interés más básico pero no el único, es recuperar la condición de ciudadano que tiene derecho a reclamar ante el estado.

Además, se propone que una red de conflictos, da posibilidades de visibilidad social a otra red de conflictos que sumergida o velada está po- 
tencialmente instalada en las relaciones sociales cotidianas.

Las redes de conflicto que se manifiestan en la protesta conforman relacionalmente un espacio multipolar de situaciones antagónicas entre actores en conflictos. Dicho espacio, que actúa como límite estructural de la protesta y como horizonte de sus génesis, se lo puede denominar campo conflictual. En el conjunto de las relaciones de atracción y rechazo que el campo genera se constituye la producción y reproducción de la protesta.

Una vez instanciados los momentos de la protesta y en el proceso mismo de su configuración los actores retoman un complejo relacional distinto para posibilitar la conformación de las salidas a la protesta, a dicho campo se lo puede nominar como campos de negociación. Al igual que el campo conflictual su configuración responde a los mecanismos de atracción y rechazo, por lo que la delimitación del campo de negociación implica estrategias de persuasión y procesos de conversación donde lo perlocusionario y lo ilocusionario no pueden distinguirse con claridad. El campo de negociación es en sí mismo un complejo relacional instalado en paralelo a las acciones disruptivas orientado a "diferenciar auditorios" y "señales".

Es en este sentido que, en el momento donde la protesta «busca su salida» se conforma un lugar, muchas veces físico, donde las discusiones en torno a las diferentes valoraciones sobre los bienes que disparan el conflicto se lleven a cabo, este lugar es elárea de neutralidad de la protesta. Lugar donde emerge la figura de arbitraje y mediación. Para salir de la dialéctica entre conflicto y negociación se hace necesario un topos más allá de las determinaciones que impone el influjo estructural.

De este modo se puede comprender como Campo conflictual, Campo de negociación y Área de neutralidad constituyen las "Condiciones de la Acción Colectiva", es decir, constituyen una pauta de interpretación de los factores que marcan y redefinen la protesta.

De todas maneras hay que tener presente siempre que este conjunto 
de supuestos que permiten ordenar el material "empírico" no aluden la protesta como un simple epifenómeno de la situación estructural, ni apelan a la espontaneidad como criterio de comprensión del fenómeno colectivo, sino más bien se orienta a rescatar el paso de mediación de la compleja relación entre la constitución de actores particulares, situación colectiva, movilización y protesta. Para complementar esta imagen del mundo de las protestas se debe ahora posibilitar la articulación entre estructura y proceso con un análisis del "ritmo" de las protestas sociales.

Como se ha afirmado las redes de conflicto son las condiciones de surgimiento de la acción colectiva implícita en la movilización que da "forma" a la protesta. Desde aquí se puede reconocer una pluralidad de actores que rivalizan en torno a intereses comunes y que de alguna manera se orientan a una redefinición de los espacios públicos de ese momento.

Las Redes de Conflictos que preceden y operan como trasfondo de las Protestas actúan en el tiempo reconvirtiendo y redefiniendo las posiciones de los agentes y el sentido de las acciones. Como se adelantara se estructuran dos campos en permanente redefinición: el campo conflictual y el campo de negociación, articulados por un área de neutralidad. Estos espacios se actualizan en tiempos, en ritmos, que adquieren por su valor específico, un peso significativo especial.

La estructuración de las acciones colectivas devenidas, protestas sociales son formas de espacialización de los tiempos en que los actores anudan metas, decisiones, inversiones emocionales y recursos expresivos. Dicha espacialización puede comprenderse si se acepta la conformación de tres momentos en la protesta: episodio, manifestación y expresión. Nace así la oportunidad conceptual de captar la acción en sus propias condiciones, es decir, poder distinguir e interpretar lo que ocurre, lo que es observado y el significado que esto implica. Para lo cual, es importante distinguir las expresiones del conflicto, la manifestación de la acción colectiva y los distintos episodios que asumen las aludidas Redes de Conflicto. 
Las expresiones del conflicto son las acciones donde se concentran la pugna de los intereses y valoraciones en juego que tienen la característica de re-orientar la red conflictual. En estas expresiones los agentes extienden o restringen las áreas de intereses afectados, recomponen las fuerzas de las alianzas que existen entre ellos, etc., por lo que estas expresiones se caracterizan por dejar una "marca" en la orientación del conflicto. Estas marcas muchas veces permanecen latentes, no dichas. Son posicionamientos y reposicionamientos de los actores que a un nivel "estructural" tienen un poder generativo de las prácticas individuales y colectivas que se observaran en los episodios.

Los episodios del conflicto son los resultados de las expresiones del conflicto que concentran públicamente las acciones de los contendientes y que se caracterizan por evidenciar la red conflictual en términos de posiciones antagónicas y de la constitución y visibilidad identitaria. Los episodios evidencian la procesualidad de la acción colectiva en torno a las redes conflictuales. La relación entre expresiones y episodios se pone de manifiesto en la delimitación del campo conflictual y de negociación, pues anudan en el espacio-tiempo la interrelación entre los actores.

Las manifestaciones son acciones colectivas resultado de la redefinición del espacio público operado entre expresiones y episodios del conflicto. Son lo que observamos como forma de la protesta, implican la o las acciones puntuales fugaces o de cierta permanencia que el colectivo que protesta muestra como mensaje de visibilidad social. En las manifestaciones se condensa y reproduce la identidad de los actores en conflicto y esta aparece como batalla por la apropiación de sentido.

Es en este contexto que la protesta puede ser conceptualizada como la interconexión de "momentos" que evidencian la relación entre expresiones, episodios y las manifestaciones que involucran una acción colectiva en el marco de una red de conflictos. En esta dirección el tiempo-espacio de una protesta deviene mensaje en si mismo y nos traslada a una compleja 
Sociologias, Porto Alegre, ano 5, no 9, jan/jun 2003, p. 64-104

urdimbre de significado.

Forma, Mensaje y Densidad Significativa se anudan en las protestas en la constitución de sus Recursos Expresivos. Una de las apuestas más fuerte de la perspectiva que aquí se sostiene se refiere al peso específico de la forma de la protesta (Scribano, 1999e). En principio la forma se asocia a: los recursos expresivos utilizados en la protesta, los modos de articulación tiempo-espacio que los agentes producen en la protesta y las relaciones simbólicas que las protestas implican en tanto construcciones de audiencias. Particularmente la noción de forma alude a la especial relación que tienen en las protestas sujetos, espacio, discurso y sentido.

Para comprender el valor de la forma se debe recurrir en primera instancia a: identificar el símbolo o los símbolos que sirven de mediación identitaria. De esta manera emerge como uno de los ejes de la protesta la utilización de recursos expresivos. Se presenta en toda protesta una disposición significante de los modos de articulación que los actores generan a la hora de "seleccionar" los recursos expresivos que utilizan.

Un recurso expresivo se puede conceptualizar como un objeto textual que permite delimitar, construir y distribuir socialmente el sentido de la acción. Los recursos expresivos se construyen y utilizan como productos de sentido y son a la vez sentido en-producción. Son recursos en tanto resultados y también como insumos. Desde la perspectiva de los insumos los recursos son seleccionados y usados resignificando su posición original en una trama de significados determinada, desde la perspectiva de resultados los recursos se ven tamizados por un proceso de producción significativa que deviene en utilización "novedosa".

En este marco se puede entender cómo los recursos expresivos manifiestan mensajes hacia el interior del colectivo, hacia los contendientes de una determinada red de conflicto, crean espacios de consenso y generan audiencias.

En el marco de los procesos de reconocimiento y heterorecono- 
cimiento los recursos expresivos devienen marcas de la identidad colectiva que los actores se dan a sí mismos en la práctica simbólica de la apropiación y producción de dichos recursos. El mensaje se orienta también hacia los contendientes que en el marco de un campo conflictual identifican mediante los mismos la direccionalidad del sujeto antagónico. Por otro lado, los recursos se utilizan para reconstruir espacios de entendimiento que potencialisen la generación de consensos. Finalmente, los recursos expresivos permiten construir audiencias donde la dialéctica visibilidad invisibilidad tiene lugar.

En tanto mediciones simbólicas que anudan en el espacio-tiempo los procesos identitarios, los recursos involucran y ubican a los sujetos colectivos justamente en esa línea muy tenue entre sistema e individuo, entre agencia y agente, entre estructura y sujeto.

La expresividad de los recursos supone las inversiones emocionales de los sujetos que conforman el colectivo, las acciones estratégicas del colectivo y las potencialidades cognitivas de la acción. Melucci quizás es quien más ha enfatizado el rol que juegan las inversiones emocionales en la constitución de las identidades colectivas. Aquí se pretende subrayar la conexión que estas apuestas emocionales tienen con la configuración de los recursos expresivos. Los actores ponen en juego en el sistema de acción que involucra la protesta una serie de apoyos afectivos que de una manera $\mathrm{u}$ otra potencian el cruce entre lo colectivo y las valoraciones que tienen lugar en la constitución del si-mismo.

En relación a los cortes de ruta se ha intentado señalar en otro lugar que en el contexto de la significatividad de la acción colectiva se ha podido observar cómo la constitución del juego visibilidad-invisibilidad opera de diversas maneras (Scribano, 1999b; 1999c). Aludiendo, de este modo, a los efectos de los procesos de identidad colectiva que los grupos viven particularmente y que se cristaliza de manera especial en lo que se denominó densidad significativa de la acción colectiva. En conexión con lo 
Sociologias, Porto Alegre, ano 5, no 9, jan/jun 2003, p. 64-104

anterior, se afirmó que las desigualdades sociales se vienen metamorfoseando, en una suerte de proceso de acumulación de situaciones de distribución diferencial e injusta de diversos recursos. Se intento, así, mostrar cómo la pluralidad de desigualdades se presenta como punto de partida para la recuperación de la ciudadanía, como plataforma del reclamo de autonomía y solidaridad.

Es en el marco del análisis de los cortes de ruta desde donde se puede entender que las protestas exceden la relación medios-fines analizada desde la eficacia de la acción. En este sentido se puede observar también que la forma y el mensaje están íntimamente ligados. Por lo que es importante enfatizar que el mensaje de la protesta se vincula a: las demandas de subjetividad por el lado de la identidad personal y las demandas de visibilidad por el lado de la identidad colectiva. Pero también el mensaje guarda una estrecha conexión con los "límites de compatibilidad del sistema" y la tarea "profética" de las protestas.

Es en este contexto donde adquiere importancia poder conceptualizar la densidad significativa de las acciones colectivas. El accionar colectivo involucra una serie de juegos entre significantes e identidades que trasciende la noción de sentido de la acción subjetiva. No es solamente la característica de "conducta agregada" lo que complejiza el análisis y comprensión del sentido de la acción colectiva sino, más bien, su opacidad significativa.

Se ha señalado en otro lugar (Scribano, 1999b), como juegan la porosidad de lo social y la estratificación del sentido de la acción en la constitución de la densidad significativa de la acción colectiva, retomemos aquí la relación entre expresividad del si-mismo, manifestación de las redes de conflicto y campo conflictual para aclarar aún más su extensión conceptual.

En las protestas los sujetos invierten en el colectivo una serie de expectativas y anhelos con sentido subjetivo que se redefinen y resignifican de acuerdo al estado del campo conflictual y que se expresan a través de 
los recursos expresivos de la manifestación particular en la que entran en juego. Así el significado de la acción colectiva supone una trama densa de significaciones donde la acción, su forma y los recursos utilizados devienen en una acción que da que pensar. Acción, discurso y sentido se anudan en una epifanía de lo que se dice en referencia a lo que se ha querido decir, lo que no se ha podido decir y lo que aún falta por decir.

Como se podrá advertir, entendiendo a un recurso expresivo como un objeto textual que permite delimitar, construir y distribuir socialmente el sentido de la acción, se puede intentar captar la acción colectiva en su capacidad simbólica, en sus facetas de constitución de densidad significativa. Esto es aún más provechoso si se piensa que los recursos expresivos se construyen y utilizan como productos de sentido y son a la vez sentido enproducción.

Luego que se han aclarado los conceptos básicos para analizar los cortes de ruta en tanto protestas, estos se deben complementar con otros que fueron emergiendo de los análisis concretos y que apuntan a permitir la interpretación de las protesta como fenómeno más general (Scribano, 2000a; 2000b; 1999b; 1999e; 1998b y 1998c). Sólo a manera de síntesis y consignado los de mayor peso, podemos señalar los siguientes:

1. Las protestas implican una señal de los límites de compatibilidad sistémica en relación a los mecanismos de resolución de conflictos. Las demandas plurales y contradictorias de las protestas constituyen en su eficacia simbólica un mensaje claro de la incapacidad de respuesta del sistema político formal ante los efectos de la acción estatal.

2. Las protestas involucran la configuración de diversas identidades en un proceso de redefinición de la esfera pública. La incapacidad del sistema político señalada anteriormente produce el desplazamiento de los agentes hacia diversos espacios públicos donde se produce una redefinición identitaria de quienes se encuentran y también de dichos espacios.

3. Los actores que protestan buscan reducir la incertidumbre que provoca 
Sociologias, Porto Alegre, ano 5, no 9, jan/jun 2003, p. 64-104

la precariedad social y la resignificación institucional. El efecto de la precariedad laboral es la contingencia permanente del agente en los momentos de coordinación de la acción, lo que lleva a los colectivos y a los sujetos a buscar espacios y momentos para reducir la incertidumbre que ello provoca.

4. Existe una multiplicación de los centros de poder que provoca un descentramiento de las protestas. Los que protestan dirigen sus reclamos simultáneamente al estado nacional, provincial y municipal, a los partidos políticos, a los medios de comunicación y a los empresarios.

5. Estamos en presencia de una redefinición de los mecanismos de coordinación de la acción en el marco de una dialéctica entre clientelismo y representación social y política.

6. Se evidencian la constitución y reconstitución de clases en trayectoria y la distancia en términos económicos provocada por la representación política. Los desocupados, los nuevos pobres, los pobres estructurales, las clases en descenso y lucha por la permanencia en el sistema, constituyen clases en trayectoria de redefinición permanente que contrastan con la consolidación de los políticos como clase social.

7. Las protestas señalan también la ausencia de una praxis social común y se postulan como referentes de un mundo que ya no es y aún no puede ser.

8. El potencial transformador de las protestas radica en las transformaciones que el protestar supone, el superar la invisibilidad, el reconstruir lazos colectivos, el resignificar tradiciones y el uso de formas expresivas para señalar los límites del sistema.

9. En las protestas se entrecruzan utopía y acción colectiva de una manera diferente, sin la apelación a un centro, sin metáforas mecanicistas desde la pluralidad que acarrea la fragmentación, superando la precariedad. Sin un telos previamente definido las protestas no son el movimiento argentino de liberación nacional, son la expresión más acabada de los 
límites de la acción en contextos de empobrecimiento y disolución de la política.

Estas opciones teóricas se entrecruzan con las epistémicas sosteniendo el diseño de una estrategia metodológica. Lo que se ha reseñado aquí muestra la interconexión entre los supuestos epistémicos, relacionalidad, indeterminación y dialéctica, con los ontológicos sujeto e identidad (sólo para mencionar algunos) y con los conceptos de la red teórica. En el próximo apartado se resumen las características fundamentales de dicha estrategia enfatizando que producimos aquí una distinción analítica para facilitar su recuperación sistemática, pero que en lo concreto del proceso de observación e interpretación se presenta como una dialéctica del comprender el mundo social.

\section{Estrategia metodologica general y tecnicas utilizadas}

En el contexto de los supuestos teóricos y epistemológicos que se han explicitado se enfrentaron conjuntamente tres tipos de desafíos metodológicos: la necesidad de observar un fenómeno que, generalmente y en apariencia, tiene la característica de aparecer muy espontáneamente, la imposibilidad de identificar a priori los agentes organizadores y participantes y el rasgo local de los eventos.

Por lo cual, se pensó en la siguiente estrategia combinada de fuentes de información y producción de datos orientada a captar la conflictividad, la estructuración temporo-espacial y los modos de expresividad que implicaban los cortes:

a) Registro, sistematización y análisis de archivos periodísticos televisivos en Internet. En este sentido generalmente se utilizaron los archivos de un canal de noticias de alcance nacional Ilamado Todo Noticias que posee una base de datos de noticias desde 1996 con buscador propio. 
Sociologias, Porto Alegre, ano 5, no 9, jan/jun 2003, p. 64-104

b) Registro, sistematización y análisis de Diarios, en soporte papel e Internet. Complementariamente con los archivos de Internet se realizaron búsquedas en periódicos locales, tales como La Unión de Catamarca, La Voz del Interior de Córdoba y El Diario de Villa María, como así también en uno de circulación nacional como El Clarín.

c) Registro, sistematización y análisis de relatos de Actores: Las vías ya mencionadas no permitían captar el punto de vista del sujeto por lo que se recurrió a: Narraciones, como en el caso de Cultra Có donde se obtuvieron 91 relatos de jóvenes de la localidad, a Entrevistas a los participantes en los cortes, ya que fueran cedidas por otros investigadores como el caso de Belén o que fueran hechas por el equipo como en el caso de Villa María, finalmente se utilizaron las declaraciones y manifestaciones de los actores aparecidas en diarios, revistas y documentos de los colectivos que participaban.

Una vez elaborados los registros mencionados se procedió a identificar y describir las protestas y a la identificación del contexto estructural de las mismas, en los siguientes términos:

\section{Identificación y Descripción de las Protestas}

a) Primero se buscaron las relaciones entre Actores, Lugar, Fecha, Demandas y Modalidad de la protesta.

b) En segundo lugar, se identificó las redes de conflictos preexistentes o convergentes.

c) Luego, se establecieron las Zonas y/o Ciudades de "cortes" y/o protestas y,

d) Finalmente, se puso en relación a) y b)

\section{Identificación del Contexto Estructural}

Con el convencimiento que se debía poner en contexto los registros obtenidos se identificaron y sistematizaron una serie de indicadores que permitieran comprender la aparición de las redes de conflicto, los más 
usados fueron los siguientes:

a) Indicadores Socioeconómicos: Distribución del Ingreso, NBI y Desocupación.

b) Indicadores Sociopolíticos: Participación Electoral y "Representatividad Regional".

c) Programas de Focalización: FOPAR, PAGV, PLAN TRABAJAR.

d) Presencia de organismos internacionales: Créditos Banco Mundial.

Las acciones descriptas posibilitaban identificar e interpretar el conflicto, la protesta y el contexto estructural pero se percibió un déficit fundamental: la visión de los sujetos, para ello se produjeron los análisis de los relatos de los actores consignados arriba. Los objetivos fundamentales fueron: a) captar la definición de la situación a través de la narración del agente; b) estructurar la base de una primera interpretación del sentido de la acción y c) buscar las representaciones de los actores que, de modo recurrente, abriera el camino para la construcción de categorías de análisis.

Ahora bien, esta estrategia de caminos múltiples configuraba un complejo de acciones donde instrumentos y procesos adquirían un sentido en tanto totalidad.

Los instrumentos se seleccionaron por su capacidad intrínseca respecto a la información que se busca relevar: El análisis del material periodístico conducía al establecimiento de las redes de conflicto, el campo conflictual y el campo de negociación. Las entrevistas individuales y los relatos, que en primera instancia posibilitan la incorporación del punto de vista del sujeto respecto a los temas aludidos, además implicaban la posibilidad de abordar los procesos de construcción de sentido y constitución identitaria. Solidario con los instrumentos anteriores el análisis de los documentos "producidos" por los actores permite completar la información "estructural" y subjetiva con los procesos de construcción de marcos de significado y contextos de consensos. La articulación entre registros de información periodística y los relatos de los sujetos permitió explorar la utilización de 
Sociologias, Porto Alegre, ano 5, no 9, jan/jun 2003, p. 64-104

los recursos expresivos.

Este tejido de información e interpretación fue dando forma a una vía estandarizada para "observar" los cortes en su particularidad, que se constituyo en la guía conceptual para la estrategia general de búsqueda, registro y análisis de información:

a) Se tomo como "guía" las conceptualizaciones de episodio, manifestación y expresión de la protesta.

b) Dado el punto de partida teórico que se explicitara, se propuso identificar tres tipos de redes de conflictos, en tanto Condiciones de la Acción Colectiva: los que hacen referencia a conflictos nacionales, los que se refieren a los conflictos puntuales o de los actores involucrados en la protesta y los regionales o locales que se conectan con la situación.

c) Se identificó y sistematizó la trama de la redes de conflictos que configuran el campo conflictual y el campo de negociación de la protesta.

d) Se analiza la situación de surgimiento, es decir, el paro, movilización o marcha que da origen a la protesta.

e) Descripto el surgimiento y poniéndolo en relación con los conflictos identificados se pasa al análisis de la profundización del conflicto, corte de ruta y utilización de otros recursos expresivos.

f) Abordadas las facetas anteriores se analizan los siguientes rasgos: su relación con el corte de la circulación, las nuevas relaciones y contactos a las que da comienzo, las formas de violencia que implica, los significados de los recursos expresivos estudiados y la relación entre ilegalidad y ciudadanía.

g) Estudiadas estas condiciones se hace posible dedicarse al problema de la visibilidad social que implican estos tipos de protestas.

h) Es en este marco que se focaliza el análisis en la Movilización por la visibilidad, confrontación contra la represión y desmovilización negociada.

i) Finalmente, se describirá la relación entre el campo conflictual y el 
campo de negociación, como articulación necesaria para analizar el momento de negociación y la selección del área de neutralidad.

Dada esta clave interpretativa creemos que se pueden también ofrecer algunas otras pistas de la utilización del esquema teórico-metodológico que hemos presentado.

Siempre teniendo presente que, como se afirmó ya, para comprender el valor de la forma de la protesta se debe recurrir en primera instancia a: identificar el símbolo o los símbolos que sirven de mediación identitaria de la misma. Y además, es necesario comprender cómo Campo conflictual, Campo de negociación y Área de neutralidad constituyen las "Condiciones de la Acción Colectiva", es decir, estas condiciones son una pauta de interpretación de los factores que marcan y redefinen la protesta.

En esta dirección, en primer lugar, hay que localizar la red conflictual, esto hace referencia directa a la trama que opera como trasfondo de la movilización; luego retomar los cortes desde lo "hechos" que le dan su primera visibilidad, es decir, paro, marcha u otra forma de acción colectiva que los precede en el tiempo y donde actores y demandas aparecen conectados por primera vez públicamente. Esto se realiza dando cuenta de las posibles conexiones entre las redes de conflictos más amplias y el momento de emergencia "más fuerte" de las redes sumergidas que ellos implican.

En el contexto de nuestras investigaciones, descripto el surgimiento de los cortes y poniéndolo en relación con los conflictos enunciados se hizo necesario pasar al análisis de la profundización del conflicto, lo que condujo directamente a la relación corte de ruta y represión. En el análisis de la represión parece necesario retomar desde su misma raíz a los "mecanismo represivos" dado que en los cortes se ha puesto de manifiesto nuevamente la disparidad entre la noción de seguridad ciudadana y las prácticas de las "fuerzas de seguridad".

En la interpretación del "corte" mismo se proponen como ejes 
Sociologias, Porto Alegre, ano 5, no 9, jan/jun 2003, p. 64-104

temáticos importantes los siguientes rasgos: su relación con el corte de la circulación, las nuevas relaciones y "contactos" a las que da comienzo, las formas de violencia que implica, la relación entre ilegalidad y ciudadanía y los recursos expresivos utilizados.

Al cortar la ruta se produce una redistribución de la apropiación de los espacios y una redefinición de las comunicaciones y con ellos aparecen delimitados los campos conflictuales y las áreas de neutralidad. El campo conflictual se construye a partir de las redes de conflictos que dan origen a la protesta y guardan una estrecha relación con lo que hemos denominado momentos del conflicto. Estos campos son las relaciones conflictuales que se establecen en el momento de la expresión del conflicto y la movilización e involucra la participación de actores particulares que pueden o no estar presentes en la red de conflicto que le da origen. De este modo, jueces, policías, camioneros, etc., pasan a formar parte de un campo conflictual en el momento de la protesta. Las áreas de neutralidad remiten al momento de des-movilización con negociación. Para levantar el corte, hay que ir a un terreno donde los poderes sociales estén, al menos parcialmente, imposibilitados de echar ha andar los mecanismos tradicionales de cooptación de líderes y de conciencias. De este modo, la definición de los "lugares" de negociación son tan importantes como los reclamos que se pretenden negociar. Además, esto se conecta con la razón de legalidad, debe ser un área que establezca un paréntesis con la ilegalidad pero que permita mantener la posibilidad de regresar a ella.

Los cortes en el mismo momento que se instauran los procesos de negociación se plantean el problema del "levantamiento". Nuestra clasificación remite a tres tipos de levantamiento: parcial, total y provisorio. El primero es un modo de llegar a la negociación, el segundo y el tercero son las alternativas posibles que surgen de los resultados de la negociación. Frente a la amenaza de represión o la represión concreta los piqueteros y fogoneros envían un mensaje más, la suspensión parcial del corte como signo de 
posibilidades de negociación, generalmente a través de la mediación de algún actor proveniente del área de neutralidad. Esto facilita las concreción de las condiciones de diálogo y comienza a modificar el campo conflictual. La negociación misma determina el alcance espacial y temporal del levantamiento, si los reclamos son atendidos "solidamente" el levantamiento es total, en cambio si existe alguna duda la modalidad de provisorio deja en manos de los piqueteros y fogoneros la respuesta al incumplimiento.

Hemos ido así de los instrumentos a las estrategias como una senda para recorrer sistemáticamente. Se ha percibido como esta visión sobre el estudio de una protesta particular como lo son los cortes de ruta implica una urdimbre interpretativa donde se articulan supuestos epistémicos, construcciones teóricas y opciones metodológicas. Ahora es conveniente preguntarse sobre las conclusiones posibles respecto a la utilización de dicha visión.

\section{Mirando al sesgo: algunas conclusiones sobre el estudio de los cortes de ruta}

Ahora bien, ¿qué podemos aprehender de este estilo de investigar protestas en general y cortes de ruta en particular? Teniendo en cuenta los objetivos de este trabajo, la respuesta debe ser efectuada, al menos, en tres niveles: el epistemológico, el teórico y el metodológico:

En primer lugar, emerge de la experiencia reseñada, la necesidad de mantenerse alertas a nivel epistemológico respecto a dos componentes del proceso de conocimiento:

1. Las protestas y los conflictos deben ser analizados partiendo de la idea que se esta enfrente a sistemas abiertos y en presencia de un mundo estructurado, estratificado y diversificado. Es decir, superar el sesgo empirista por el cual se tiene la tendencia a mirar la protesta desde la reificación de los agentes y sus relaciones como un dato dado con ras- 
Sociologias, Porto Alegre, ano 5, no 9, jan/jun 2003, p. 64-104

gos inmutables. Más allá que por propia opción, se halla partido de un realismo "cualificado", que aplicado al análisis de las protestas debe suponer una compresión procesual y relacional del conflicto, los estudios empíricos y la búsquedas de los procesos nos han indicado la importancia de mantener dicha opción.

2. Otro elemento que surge recurrentemente es la necesidad de buscar y comenzar con el análisis de la falla, de aquello que no cierra. Es un camino que va desde la ausencia en el entramado social, y pasa por la identificación del cemento que hace posible la coordinación de la acción y llega hasta la identificación de los ladrillos, de las piezas, por las cuales se construye el entero edificio social. La ausencia detectada en la protesta como un proceso en producción dispara la posibilidad de entrever las suturas por donde se invierte el conjunto de determinaciones reales de dicho proceso.

Desde un punto de vista teórico, es posible entrever la importancia de los siguientes elementos:

1. Hay que objetivar el hecho que los análisis realizados son una construcción de la construcción. Emerge aquí la necesidad de contar con herramental teórico que posibilite rupturar y conquistar el punto de partida de muchos de los estudios sobre protestas, a saber, el discurso periodístico y la pluralización de interpretaciones del mundo que este media. En esta dirección, una vez más, se deberá estar alerta ante el desafió de captar las mediaciones necesarias para la construcción interpretativa.

2. En los estudios realizados emerge con fuerza la centralidad de captar el paso de la lectura primera que implica la visión de los sujetos y la coconstrucción textual que involucra el documento sociológico que nosotros construimos. En un juego entre el texto que elaboramos y el texto que los agentes portan se percibe el camino para actualizar las marcas de los procesos, de las ausencias y de las suturas que produce el 
entramado social.

3. En conexión con lo anterior, de los estudios que se han realizado y en el marco del interés por captar el proceso de procesos, se hace evidente que no todo se desvanece en el aire. Lo sólido aparece en este componente procesual e indeterminístico. Las múltiples determinaciones de los fenómenos se pueden encontrar si se tienen instrumentos analíticos para hacerse de su descentramiento y evanescencia. Esto es un contra argumento respecto a quiénes solo ven lo efímero de las protestas y su "inutilidad" política. Como así también para aquellos que niegan, casi por un prejuicio empirista, la posibilidad de su conocimiento más allá de ese rasgo efímero.

Finalmente, desde una perspectiva metodológica se pueden subrayar tres elementos críticos al estudio de la protesta:

1. En primer lugar, aparece como problemática la relación entre datos y registros periodísticos, que se podría sintetizar con el refrán popular, lo barato sale caro. Es decir, las más de las veces la selección de esta fuente de información se realiza por que es fácil y barato su manejo, por que permite construir cronologías y por que permite captar e identificar expost actores y acciones. El inconveniente es que si el investigador no esta alerta a que estos registros pueden ser incompletos y fragmentarios la noticia reifica el fenómeno y desplaza la atención del análisis. Por lo que es necesario poner siempre en duda, al menos provisoriamente, dichos registros. Es conveniente evaluar el precio que hay que pagar por una identificación y sistematización sencilla de los fenómenos en orden a la interpretación que se proponga realizar.

2. En segundo lugar, emerge la dificultad de establecer pautas adecuadas para la selección de indicadores estructurales, lo cual puede ser sintetizado, con el refrán popular, pobre pero no tanto. Al intentar contextualizar los conflictos, el obstáculo que se presenta es el de disponer de criterios homogéneos para aplicar a información elabora- 
Sociologias, Porto Alegre, ano 5, no 9, jan/jun 2003, p. 64-104

da por otros y cuyos supuestos y técnicas de construcción varían enormemente. En este sentido, hay que estar dispuesto a aceptar que la riqueza o pobreza de esto indicadores como señas de la estructuración social es relativa. Como así también, permanecer abiertos a la aplicación de la máxima metodológica de objetivar lo objetivado.

3. En tercer lugar, una consecuencia metodológica de los trabajos realizados es la de estar preparados para repensar nuestra propia práctica de investigación, lo cual podría ser subsumido en la frase, a científico regalado no se le miran los dientes. En el transcurso de la investigación de un tópico tan sensible, política y socialmente, como lo es el de la protesta, se aprende mucho de los propios actores y de las relaciones que el propio investigador va teniendo. El estar predispuestos a confrontar con el decurso de dichas prácticas previne de dogmatismos escolásticos. El estar dispuestos a mantener una actitud científica no nos debe dejar pasar la oportunidad de aprehender de nuestra creatividad y de la de los actores, que con el tiempo se transforman en compañeros de ruta en el interés por conocer la realidad social.

Luego de este largo recorrido por los senderos de una de las tantas formas de afrontar el análisis de las protestas sociales en general y de los cortes de ruta en particular sólo cabe volver al comienzo. La necesidad de conocer científicamente los modos de estructuración social que se cristalizan en las protestas y en los conflictos que estas implican, no es una mera demanda académica, es una urgencia. Esto desde el punto de vista de construir unas ciencias sociales reconectadas con los procesos emancipatorios debe hacernos, siempre, repensar nuestras prácticas de investigación.

\section{Referencias}

BHASKAR, R. The possibility of naturalism. Sussex: The Harverster Pres., 1979. 
Sociologias, Porto Alegre, ano 5, no 9, jan/jun 2003, p. 64-104

BHASKAR, R. Scientific realism e human emancipation. London: Verso, 1986.

COLLIER, A. Scientific realism and socialist thougth. USA: Harvester Wheatshef., 1989.

DIANI, M. y EYERMAN, R. (Edit.) Studying collective action. London: Sage, 1992.

FERNANDEZ, A. Movimientos sociales en América Latina. Bs. As.: Aique Grupo Editor, 1991.

GARRETON, M. A. Movimientos sociales y procesos de democratización. Un marco analítico. En: Excerpta, n. 2, abril, p. 1-9 (internet), 1996.

JELIN, E. Movimientos sociales y democracia emergente. Bs. As.: CEAL, v. 1 y 2. , 1987.

JESSOP, B. Social order, reform and revolution. London: MacMillian, 1972.

MELUCCI, A. Sistema politico, partiti e movimenti sociali. Milano: Feltrinelli, 1977.

MELUCCl, A. L'invenzione del presente. Bologna: Il Mulino, 1982 (1991).

MELUCCI, A. Altri codici. Bologna: Il Mulino, 1984.

MELUCCI, A. Sul coinvolgimento individuale nell'azione colletiva. Rassegna Italiana di Sociologia, XXVII, n. 1, genn.-marzo, 1987, p. 29-53.

MELUCCl, A. Nomads of the present. London: Hutchinson Radius, 1989.

MELUCCl, A. Il Gioco dell'io. Milano: Feltrinelli, 1991.

MELUCCI, A. Liberation or Meaning? Social Movements, Culture and Democracy. Development and Change. V. 23, n. 3, p. 43-77. London: Sage, 1992a.

MELUCCI, A. Frontier Land: collective action between actors and systems. In: Studying collective action. Diani, M. and Eyerman, R. (edit.). London: Sage, 1992b, p. 238-258. 
Sociologias, Porto Alegre, ano 5, no 9, jan/jun 2003, p. 64-104

MELUCCI, A. Passaggio d'epoca. Milano: Feltrinelli, 1994a.

MELUCCI, A. ¿Qué hay de nuevo en los nuevos movimientos sociales?. In: Laraña, E. and Gusfield, J. (edit.) Los nuevos movimientos sociales. Madrid: CIS/Academia, $1994 b$.

MELUCCI, A. Individualitation and globalization: new frontiers for collective action and personal identity. In: Hitotsubashi Journal of Social Studies, v. 27, August, Tokyo. 1995.

NEW, C. Sociology and the case for realism. En: Sociological Review. Blackwell Publishers, 1995.

OBERSCHALL, A. Theories of social conflict. En: Ann. Rev. Sociol. 4, 1978, p. 291 315

OUTHWAITE, W. New philosophy of social science. New York: St. Martin's Press, 1987.

SAYER, A. Method in social science: a realist approach. 2nd Edition. London: Routledge. 1992.

SCHUSTER, F. Protestas sociales en la Argentina: 1989-1990. En: Informe Anual 1996 del Centro de Estudios Legales y Sociales, Buenos Aires, 1996.

SCRIBANO, A. De gures, profetas e ingenieros: ensayos de sociología y filosofía. Córdoba: Edit. Copiar, 2002, 153 pag.

SCRIBANO, A. Identidades fragmentarias y ciudadanías nómadas. Taller: Globalización y nuevas ciudadanías. Reigadas y Cullean coord., 2000a, m/s.

SCRIBANO, A. Política, Protesta y Movimientos Sociales en la Argentina. Taller: Movimientos Sociales y Representación Política, IV Jornadas de Sociología, Universidad de Buenos Aires, Facultad de Ciencias Sociales, Preparatorias del III Encuentro por un Nuevo Pensamiento de CTA, Instituto Gino Germani Lunes 6 de Noviembre, 2000. 2000b.

SCRIBANO, A. La Diferencia Su importancia en los procesos de construcción de la Identidad personal. En: Red de Filosofía y Teoría Social, Quinto Encuentro. Centro Editor de la Secretaría de Ciencia y Tecnología de la Universidad Nacional de 
Catamarca. En proceso de Edición, 1999a.

SCRIBANO, A. Argentina Cortada: "Cortes de Ruta" y Visibilidad Social en el Contexto del Ajuste. En: Lucha popular, democracia, neoliberalismo: protesta popular en América Latina en los Años del ajuste. Venezuela: Margarita López Maya Editora/ Nueva Visión., 1999b, p. 45-71.

SCRIBANO, A. Multiculturalismo, Teoría Social y Contexto Latinoamericano. Rev. La Factoría, n. 9, Junio-Septiembre. España. www.lafactoriaweb.com/articulos/ scribano9, 1999c.

SCRIBANO, A. Epistemología y Estudios de Acción Colectiva: X Congreso Nacional de Filosofía. AFRA. Asociación Filosófica Argentina. Escuela de Filosofía, Facultad de Filosofía y Humanidades. Huerta Grande, Córdoba 24 al 27 de Noviembre, 1999d.

SCRIBANO, A. Lo que el viento se llevo: protesta social, indeterminación y sentido. Encuentro Ciencias Sociales en Argentina. Investigación y Teoría. 27 de Agosto Universidad Nacional de Villa María, 1999e.

SCRIBANO, A. Complex Society and social theory. Social Science Information, 37 (3). Sage, Sept, 1998a, p. 493-532.

SCRIBANO, A. Los otros, nosotros y ellos: hacia una caracterización de las prácticas políticas en contextos de exclusión. Fidel Molina Luque (Edit.). En: Educación, Cultura y Política. España: Servicio de Publicaciones de la Universidad de Lleida. En prensa, 1998b.

SCRIBANO, A. De la voz al espacio: los cortes de ruta y derechos humanos. En: Red de Filosofía y Teoría Social, Cuarto Encuentro. Centro Editor de la Secretaría de Ciencia y Tecnología de la Universidad Nacional de Catamarca. En prensa, 1998c.

SEOANE, J. y TADDEI, E. (Comp.) Resistencias mundiales: de Seattle a Porto Alegre. Bs.As.: CLACSO, 2001.

TILLY, C. Revolution and collective action. En: Greenstein, F. y Polsby, N. (Edit.) Macropolitical Theory, 1975, p. 483-555. 
Sociologias, Porto Alegre, ano 5, no 9, jan/jun 2003, p. 64-104

\section{Resumen}

¿Cómo identificar, entender y explicar el sentido de las acciones colectivas? Es tal vez hoy una de las preguntas más desafiantes y conflictivas para la Teoría Social en general y la Metodología de las Ciencias Sociales en particular.

Desde una perspectiva teórica son diversos los caminos disponibles para interpretar las acciones colectivas y muchas de ellas implican la opción de una específica estrategia metodológica. En esta dirección, al intentar construir un diseño de investigación para el análisis empírico de las protestas sociales se hace evidente, de forma inmediata, algunas de las dificultades básicas de la producción de conocimiento en ciencias sociales. Una de las facetas del desafío aludido la constituye la necesidad de disponer de una articulación critica entre supuestos teóricos, epistémicos y metodológicos.

Este trabajo tiene por objetivo mostrar y discutir una de las estrategias usada para investigar "Cortes de Ruta" en la Argentina en tanto forma particular de protesta social explicitando sus supuestos.

Para lograr el objetivo planteado se ha seleccionado la siguiente camino argumentativo: a) sintetizar los supuestos epistémicos básicos del abordaje utilizado; b) reseñar el instrumental teórico construido; c) describir las técnicas de obtención de información y d) reflexionar respecto a las dificultades encontradas y a las potencialidades detectadas.

Palabras-claves: protestas, supuestos epistemicos, relacionalidad, visibilidad, identidad. 